\title{
City of Things: Enabling Resource Provisioning in Smart Cities
}

\author{
José Santos, Student Member, IEEE, Thomas Vanhove, Student Member, IEEE, Merlijn Sebrechts, Student \\ Member, IEEE, Thomas Dupont, Wannes Kerckhove, Bart Braem, Member, IEEE, \\ Gregory Van Seghbroeck, Member, IEEE, Tim Wauters, Member, IEEE, Philip Leroux, Member, IEEE, \\ Steven Latré, Member, IEEE, Bruno Volckaert, Member, IEEE, and Filip De Turck, Senior Member, IEEE
}

\begin{abstract}
In the last years, traffic over wireless networks has been increasing exponentially due to the impact of Internet of Things (IoT). IoT is transforming a wide range of services in different domains of urban life, such as environmental monitoring, home automation and public transportation. The so-called Smart City applications will introduce a set of stringent requirements, such as low latency and high mobility, since services must be allocated and instantiated on-demand simultaneously close to multiple devices at different locations. Efficient resource provisioning functionalities are needed to address these demanding constraints introduced by Smart City applications while minimizing resource costs and maximizing Quality of Service $(\mathrm{QoS})$. In this article, the City of Things (CoT) framework is presented, which provides not only data collection and analysis functionalities but also automated resource provisioning mechanisms for future Smart City applications. CoT is deployed as a Smart City testbed in Antwerp (Belgium) that allows researchers and developers to easily setup and validate IoT experiments. A Smart City use case based on Air Quality Monitoring through the deployment of air quality sensors in moving cars has been presented showing the full applicability of the CoT framework for a flexible and scalable resource provisioning in the Smart City ecosystem.
\end{abstract}

Index Terms-Smart Cities, IoT, Resource Provisioning, Big Data;

\section{INTRODUCTION}

$\mathbf{I}$ $\mathrm{N}$ recent years, the Internet of Things (IoT) has introduced a whole new set of challenges in Telecommunications by transforming objects of everyday life in communicating devices [1]. With the advent of IoT, the concept of a Smart City has become even more popular [2]. IoT is transforming a wide range of services in different domains of urban life, by developing home automation applications, improving public transportation and creating intelligent smart grid systems. By 2021, the number of connected devices will be between 10 and 12 billion [3], which current network architectures will not be able to support due to the demanding constraints introduced by IoT. In fact, Smart City applications will introduce a set of stringent requirements, such as low latency and high mobility, since resources can be requested on-demand simultaneously by different devices at multiple locations. It is thus necessary to design and develop new management functionalities to help meet the strict requirements introduced by future use cases.

Authors are with Ghent University - imec, IDLab, Department of Information Technology, Belgium. Email: josepedro.pereiradossantos@UGent.be

Manuscript received December 15, 2017
Currently, there is still a large number of research challenges associated with the resource provisioning of Smart City applications. One of the challenges is how to set up the infrastructure to cope with all the data that needs to be received from the IoT devices. Traditional processing and storage solutions no longer suffice for big datasets. Large Industries and Institutions may have the funds required to host such an infrastructure, however that is not the case for individuals and smaller companies. Infrastructure-as-a-Service (IaaS) providers such as Google, Amazon and Microsoft have already partly bridged this gap by providing infrastructure on a pay-per-use basis. As datasets continue to grow however, these costs may prove too big for companies and researchers. On the other hand, developers face multiple complex technology decisions in order to select the best fit to deploy their IoT solution. Multiple data storage and analysis frameworks are currently available in the literature. Without up-to-date knowhow or experience, issues arise when developers need to make technology decisions. Moreover, one of the main challenges that still remains is how to provide proper resource allocation mechanisms for Smart City applications, since services can be placed in a highly congested location, which would result in a higher communication latency [4]. In the literature, few resource provisioning strategies are currently addressing the stringent requirements of Smart City applications while minimizing resource costs and maximizing Quality of Service (QoS) [4], [5]. Therefore, efficient resource allocation strategies are needed to address these issues.

In this article, the City of Things (CoT) framework is presented, which provides not only data collection and analysis functionalities but also enables automated resource provisioning operations for future Smart City applications. The CoT framework has been deployed within a Smart City testbed in Antwerp, Belgium [6] and is based on the Tengu platform [7], an automated orchestration service for designing big data frameworks and on LimeDS [8], a toolkit designed for the rapid development of data-driven services. Both technologies provide scalable and flexible resource allocation operations, which allow researchers and developers to design and deploy specific IoT service models. Finally, a Smart City scenario based on an Air Quality Monitoring use case is presented showing the full applicability of the CoT framework.

The remainder of the article is organized as follows. In the next Section, related work is discussed. Section III introduces the proposed CoT framework for the resource provisioning of 
Smart City applications. In Section IV, the use case scenario is described. Finally, conclusions are presented in Section VI.

\section{RELATED WORK}

In recent years, research efforts have been carried out to deal with management and resource allocation issues in Smart Cities. For example, the Smart Transportation project from the University of Toronto [9] based on connected vehicles has been investigating how effectively a vehicular network can be used to monitor and control the traffic in a Smart City. The SmartSantander project [5] has been working on a suitable architecture for the resource provisioning of IoT applications in Smart Cities. The SmartSantander framework provides a large scale testbed for experimentation and evaluation of IoT use cases in several urban scenarios. Moreover, the CityPulse project [10] has been developing an analytics framework for Smart Cities, which integrates powerful data aggregation and analysis tools, event detection modules and quality assessment algorithms, aiming to support the deployment of Smart City use cases. Furthermore, the SusCity project [11] has been designing an IoT framework focusing on data collection in order to develop management solutions for Smart Cities. However, their approach is only focused on Big Data services for Smart City use cases while the CoT approach presented here is not only concerned with data monitoring and analytics operations but also resource allocation functionalities that can help to autonomously orchestrate Smart City applications.

The SLICENET project [12] aims to maximize the potential of the future $5 \mathrm{G}$ infrastructures and their services based on cognitive network management in $5 \mathrm{G}$ networks. One evaluation scenario considered by SLICENET is a Smart City use case, aiming to implement a remote water metering and an intelligent public lighting system in the city of Alba Iulia, in Romania. Finally, The VITAL project [13] has been working on heterogeneous Smart City platforms via semantics in a cloud-based federation environment. Their goal is to develop a technology-agnostic architecture, where the integration and deployment of multiple IoT devices can be considered by neglecting the underlying architecture. All these solutions are fully compatible with the CoT testbed and could, in the future, be benchmarked on the CoT experimental platform.

Although the cited existing and ongoing research projects address the requirements of management frameworks for Smart Cities, they have not yet delivered a fully flexible and autonomous solution. Furthermore, the described Smart City testbeds are often small-scale both in terms of device count (only a few tens) and in terms of geographic location (often only a single building or a car parking area). Second, most testbeds are very homogeneously focusing on only one wireless technology (e.g. only ZigBee or WiFi). The CoT framework goes beyond the current state-of-the-art by introducing a flexible and efficient manner of dealing with all the data generated in Smart Cities, by combining data collection and analysis operations for a proper resource provisioning of Smart City applications and by supporting multiple Low Power Wide Area Network (LPWAN) technologies to accommodate a wide range of heterogeneous devices. By eliminating technical barriers, the CoT framework paves the way for researchers and stakeholders to create new applications and services in the Smart City ecosystem.

\section{The City of Things Framework}

In this section, the CoT framework is presented. First, a high-level architecture overview is discussed. Second, the LPWAN infrastructure of CoT is introduced. Third, a detailed overview of the CoT architecture is illustrated. Then, the Data Collection and Aggregation Platform is detailed. Finally, the Data Analysis Platform is presented.

The CoT project is a cross-technology testbed platform which validates key Smart Cities Research results and facilitates innovative Smart City experiments on top of a large-scale testbed environment. The CoT project aims to achieve four important goals:

1) Testing new technologies: by rolling out IoT devices across the entire city, CoT provides an ideal and realistic testing environment for new network technologies.

2) Big data platform: the project provides a data platform for monitoring life in Antwerp in real-time. It aims to turn these data streams into valuable information, which can in turn be used by new applications and services.

3) Citizen engagement: CoT leverages interactive user research allowing citizens to give feedback on the applications and services.

4) Resource provisioning: the project aims to achieve an efficient resource provisioning for Smart City applications by providing flexible data collection and analytics toolkits.

To accomplish all these objectives, the CoT testbed has created an environment in which researchers and stakeholders can use the functionalities provided by the CoT framework to fully extract useful information from the gathered data.

\section{A. Architecture Overview}

In Fig. 1, an overview of the CoT framework is shown. CoT offers researchers and stakeholders a city-wide framework to take the first important steps towards a true Smart City ecosystem. Nowadays, low power wireless technologies have gained tremendous emphasis due to the massive growth of connected devices in the network. The need for connecting simple IoT devices, such as sensors and actuators, is increasing rapidly. Currently over 100 sensors are deployed in Antwerp with a thousand more planned in the near future. Furthermore, at present, these sensors can communicate with the CoT infrastructure via three different LPWAN technologies: LoRaWAN, SigFox and DASH7 [6]. Multiple LPWANs are planned to be deployed and then assessed in the CoT testbed in order to identify the most adequate LPWAN communication enabler for each considered Smart City application.

\section{B. Low Power Wide Area Network Infrastructure}

CoT has been rolled out as an IoT infrastructure consisting of wireless gateways and sensors across the city of Antwerp, which can be used for experimentation by researchers. CoT 


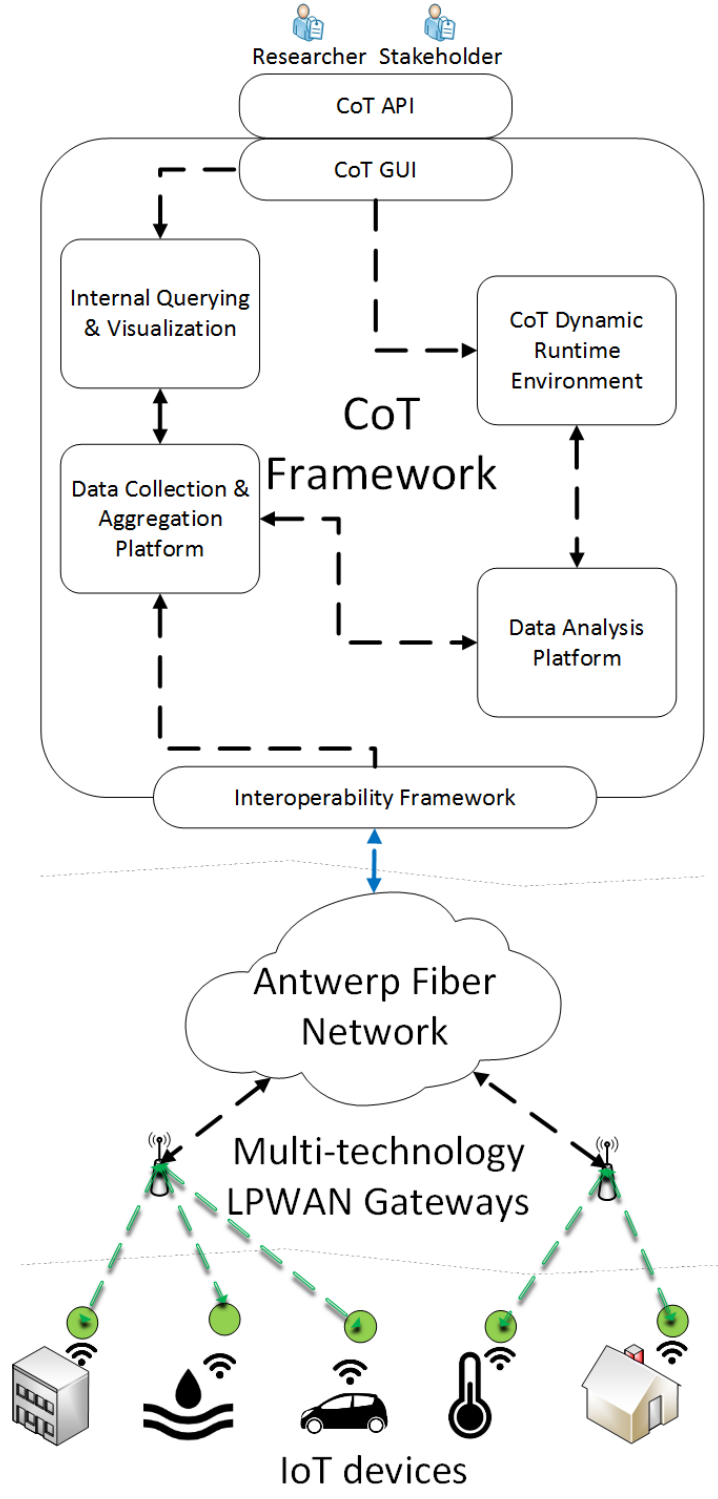

Fig. 1: High-level architecture of the CoT framework.

Infrastructure has been setup collaboratively by the IT department of Antwerp, University of Antwerp and Ghent University. The biggest infrastructural capacity within CoT is a set of hundred multi-technology gateways, which have been specifically designed and manufactured for CoT and deployed throughout the City. Each gateway has been connected to Antwerp's fiber network, which acts as a control network to provide experiment management [6]. The CoT interoperability framework is part of this control network, being mainly responsible for sending the data gathered by sensors to the Data Collection and Aggregation Platform.

Currently, multiple LPWAN technologies are available in the literature. To select a suitable LPWAN technology for a specific Smart City application, an analysis of its requirements in terms of specific parameters such as communication range, upload and download data rate, frequency bands and latency is needed. Therefore, in the CoT testbed, all deployed gateways possess a wide range of LPWAN technologies so that rapid prototyping and experimentation can be carried out, allowing the setup of multi-technology experiments and the study of the co-existence of different LPWAN technologies. Specifically, the multi-technology gateways have the following radios onboard as dedicated Systems on Chip: IEEE 802.1ac on 2.4 and $5 \mathrm{Ghz}$, DASH7 on 433 and $868 \mathrm{Mhz}$, Bluetooth (Low Energy), IEEE 802.15.4, IEEE 802.15.4g and LoRa [6]. This allows connecting both high power sensors at close range and low power sensors at long range. Moreover, other technologies, such as IEEE 802.11ah and cellular, are planned to be integrated in the future. Additionally, each gateway is equipped with a small-form-factor computer, which acts as a controller of the different radios and has ample storage and processing power available for deploying large Smart City applications directly on top of the gateways.

Although CoT multi-technology gateways provide rapid prototyping and fast wireless experimentation, each technology by itself cannot yet provide full coverage to connect all sensors city-wide. Therefore, CoT also features a separate LoRaWAN-based network, mainly used to ensure a continuous real-time stream of data from the sensors at a citywide coverage. LoRaWAN is a novel LPWAN technology, specifically intended for low-powered devices. It targets key requirements of IoT use cases, such as secure bi-directional transmissions, high mobility and long range communications. One single gateway provides a maximum communication range of 5 kilometers (urban area) and 15 kilometers (rural area) and also supports data rates of up to $50 \mathrm{kbps}$.

A large number of sensors have already been installed throughout the City for experimental validation of the CoT framework. The majority of sensors have two radios installed: a LoRaWAN radio and an additional one, which is one of the technologies already integrated within the CoT multitechnology gateways. The LoRaWAN radio is used to continuously send updates about the sensor measurements to the CoT framework, which can then be used for data collection and analysis operations. The other LPWAN technology is used to directly link the sensor to the CoT gateways and allows interaction between multiple sensors during experiments.

The following sensors are already installed in Antwerp:

1) Air Quality Monitoring: sensors have been installed on moving cars to monitor the environment, especially gas levels and temperature. More information about this use case is given in Section IV.

2) Bicycle Monitoring: sensors have been installed on bicycles to collect contextual information about the City in real-time (location tracker, event-based triggers, etc).

3) Smart Signs: smart parking signs have been used to temporarily prohibit car parking in a particular zone of the City. The signs contain an accelerometer and a GPS sensor to monitor their location.

4) Traffic Monitoring: sensors have been installed at known traffic bottlenecks in the City to monitor traffic levels based on Bluetooth and WiFi signal scanning.

5) Car Parking: sensors have been deployed in parking areas to measure their occupancy rate. 


\section{Data Collection and Aggregation Platform}

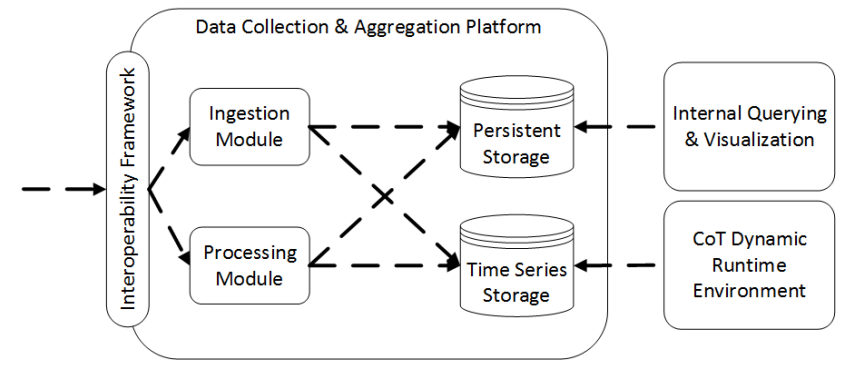

Fig. 2: Detailed view of the Data Collection and Aggregation environment in the CoT Framework.

In Fig. 2, a detailed view of the Data Collection and Aggregation Platform of the CoT framework is shown. The Interoperability framework forwards all data samples to an Ingestion module or a Processing module. On one hand, the Ingestion module handles the pre-processing of the data samples and then routes them to the Persistent Storage. Specifically, data samples are formatted and routed to the best fitting storage technology in order to make responses to the Application Programming Interface (API) requests as easy as possible. On the other hand, the Processing module provides advanced analysis modules for the incoming data samples from which the processed data can also be routed to the Persistent Storage. There are two different types of storage in the platform: Persistent Storage, which contains events, metadata and context, and a Time Series Storage solution. Both storage types are used for two different endpoints. The first endpoint is the Internal Querying and Visualization tool. It is used to monitor the environment and provide insights into all data flowing through the system. The second endpoint is the CoT Dynamic Runtime Environment, which is the access point for all researchers and stakeholders through an API that provides commands to access the available data. Additionally, the CoT Dynamic Runtime Environment can retrieve datasets for researchers, which they have access to, but also engage with sensors and actuators in the urban environment in order to test novel functionalities.

The API that relays the commands to the CoT Dynamic Runtime Environment has four entry points: sources, types, locations, and labels. The sources-API holds all the data on the available sensors, their details and the actual events of these sensors. The types-API grants access to data on the different types of sensors and which of the sources are of which type. Moreover, the locations-API provide events that are originated at a certain location. Finally, the labels-API is a simple grouping for all types/sources that are part of a certain experiment or a particular use case. A conscious technical decision has been made to only allow polling on the API, contrary to the subscriber-based streaming support used in other frameworks as in the SmartSantander testbed. The advantage of the polling-only approach is that the API becomes more scalable because unilateral urls are mapped to the different entry points, allowing the deployment of efficient caching strategies. Furthermore, the polling-only restriction is mitigated by the Data Analysis Platform, detailed next.

\section{Data Analysis Platform}

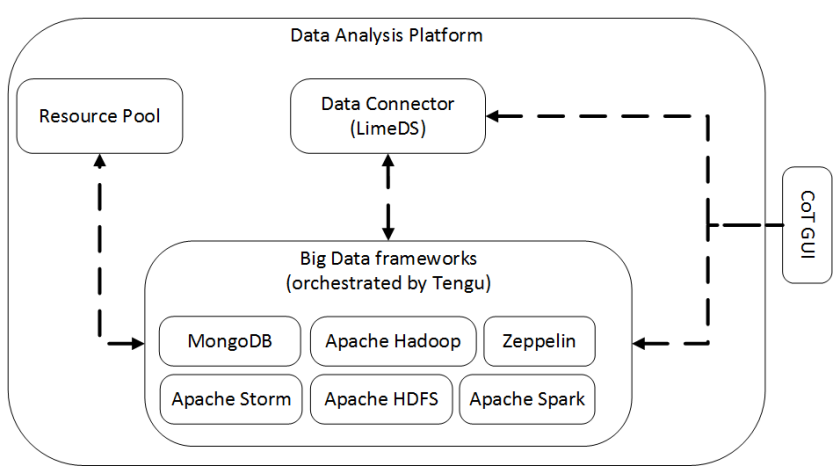

Fig. 3: Detailed view of the Data Analysis Platform in the CoT Framework.

In Fig. 3, the Data Analysis Platform of the CoT framework is illustrated. This environment is managed by the Tengu platform, which is a Platform-as-a-Service developed at Ghent University to orchestrate the setup of Big Data frameworks. Tengu manages the entire Data Backend of the CoT framework as a resource pool enabling the necessary flexibility to quickly provision custom big data frameworks in the Data Analysis Platform when needed. Tengu provides predefined bundles for specific services, which help researchers in choosing the relevant technologies for their custom applications. Researchers can connect to the Data Analysis Platform through the CoT Graphical User Interface (GUI) from which they are able to build their custom Big Data solutions. As soon as the custom Big Data framework is designed, resources are provisioned by the Tengu platform. These resources can originate from public cloud providers such as Google, Amazon and Microsoft or can be part of a private infrastructure. The applications and services created by researchers are then installed and configured on these resources. All provisioning operations are completely automated by the Tengu platform eliminating the need for manual interventions during the setup and configuration processes. Therefore, Tengu provides scalable and flexible resource provisioning operations, which allow the custom design and deployment of specific data service models.

The data feeding from the Collection and Aggregation platform is realized through an advanced data connector, supported by LimeDS. LimeDS started as an OSGi abstraction layer, but then evolved to a visual toolkit to quickly wire data-driven services together. LimeDS supports the creation of new data flows and modifications to existing ones at runtime, allowing Tengu to configure custom flows for the Data Analysis platform. Furthermore, LimeDS can create custom APIs so that applications can form several requests and combine data events to get the complete information due to the strict unilateral URLs supported by the standard API introduced in Section III-C for scalability purposes. An example of a custom API is for REstore [14]. The custom API contains prepared data for the highly specialized REstore analytics platform aimed at smart power control.

As mentioned in Section III-C, the Data Analysis platform is able to mitigate the polling-only restrictions on the CoT 
API. Subscription-like services can be set up with the available streaming technologies in the framework, which can be relevant for researchers and companies testing new sensors in the City. A streaming solution allows them to quickly follow up on early data events from the sensors and intervene, if necessary. Data events are gathered by the sensors and sent to the Interoperability framework that routes the data events through the Ingestion module into a Time Series Data Storage, such as InfluxDB. As soon as data events arrive in InfluxDB, Tengu provisions the necessary resources for the needed data services, for example Apache Spark services for micro-batch analysis. Examples of analysis operations are prediction algorithms, trend analysis and anomaly detection mechanisms. LimeDS will request the data events through the CoT Dynamic Runtime Environment and, then, these data events are transferred to the Hadoop Distributed FileSystem (HDFS), where the Apache Spark instances previously allocated are executed. After the completion of the analysis operations, Zeppelin is provisioned by Tengu allowing the visualization of the results.

Moreover, an important aspect that has not been mentioned so far is the structure of the data accessible through the CoT platform. Currently, the data is delivered as is to the external APIs and to the Data Analysis platform. However, developers might want to use specific data storage technologies with characteristics matching the requirements of their use cases. Due to the large amount of different storage technologies, it is currently left to the responsibility of the developer to transform the data into his preferred format. Nevertheless, research has been performed towards the automated transformation of both schema and data between different storage technologies. The algorithm proposed in [15] detects the format of the dataset from the original data storage and transforms it in such a way that it can benefit from the characteristics of the target data storage technology, allowing the data to be presented in any supported technology of their choice. This transformation operation is performed by the data analysis platform.

In summary, the Data Analysis platform of the CoT framework provides a flexible manner of dealing with the challenges related to the resource provisioning of Smart City applications. Both Tengu and LimeDS provide efficient resource provisioning mechanisms by allowing researchers to design and deploy specific service models in a completely automated manner. By eliminating technical barriers and the need for manual interventions, the CoT framework paves the way for developers to create specific IoT service models in the Smart City ecosystem.

\section{Use CASE - AIR QuAlity MONitoring}

As an initial proof of concept of the CoT framework, air quality sensors have been integrated in collaboration with the Belgian postal services Bpost. For daily mail delivery, Bpost has cars driving around in the city of Antwerp. A set of air quality sensors have been mounted on the roofs of Bpost's delivery cars as shown in Fig. ??. These sensors send measurements at regular intervals of typical gases and climate data, such as temperature and humidity, which are then annotated with GPS locations. As each Bpost car is continuously driving around in the city, the set of sensors can cover the entire city in terms of measurements allowing the gathering of real-time air quality information with broad city coverage, as opposed to an approach with static sensors, which only allow the gathering of limited local information. Furthermore, the number of static sensors necessary to cover the entire city is huge when compared to the needed number of cars and, thus, the installation and maintenance costs are also higher, which represent a considerable restriction when extending this kind of deployments.

The objective of this use case is to show the current status of the environment in the city of Antwerp and to detect high amounts of organic compounds in the atmosphere and then alert citizens of air pollution in real-time. With these data samples available in the platform it is interesting to create a trend analysis on the evolution of air quality in the City and perform anomaly detection operations. The data samples are gathered by the sensors and then sent through the multitechnology gateways deployed throughout the City. Then, the Interoperability framework forwards the data samples to the Ingestion module. Via the ingestion module, the measured events are stored in a Time Series Data Storage, such as InfluxDB. In order to calculate the evolution of air quality in a specific location for a certain time frame, Tengu provisions a specific data service for micro-batch analysis instantiated with Apache Spark. LimeDS will request the relevant data events in the given time frame from the CoT Dynamic Runtime Environment. Then, these data events are transferred to the HDFS, where Apache Spark algorithms are executed. Then, outlier detection algorithms have been used to identify unusual events or abnormal patterns in the dataset, such as Robust Covariance (RC) and Isolation Forrest (IF). Outlier detection is related to the identification of unusual data samples when compared to the rest of the dataset. Outliers must be further analyzed by application experts in order to extract more information from them. This way, the outlier detection outcomes have been compared with the correspondent GPS locations to know exactly where in the city of Antwerp each sample has been measured. Finally, on top of Apache Spark, Zeppelin has been integrated allowing the visualization of results. In Fig. 5, the location of two different Bpost cars where air quality data has been measured during their delivery routes is shown. Moreover, outliers detected by the $\mathrm{RC}$ algorithm for the three Particle Matter Indicators (PM1, PM2.5 and PM10) collected are shown. The outliers can be explained by high values collected of PM1, PM2.5 and PM10, which can be related to high traffic volumes in the City at these locations at the time of the measurements. By conducting anomaly detection operations in the CoT framework, citizens can be alerted of high air pollution levels in near real-time.

The Air Quality Monitoring use case shows the strength of the CoT platform and the full framework applicability in the Smart City ecosystem. Although the presented scenario is not entirely a Big Data use case, due to the fact that the available dataset is reduced, it provides a complete proof of concept of the CoT framework. CoT is an experimentation platform focused on a wide range of wireless technologies allowing the 


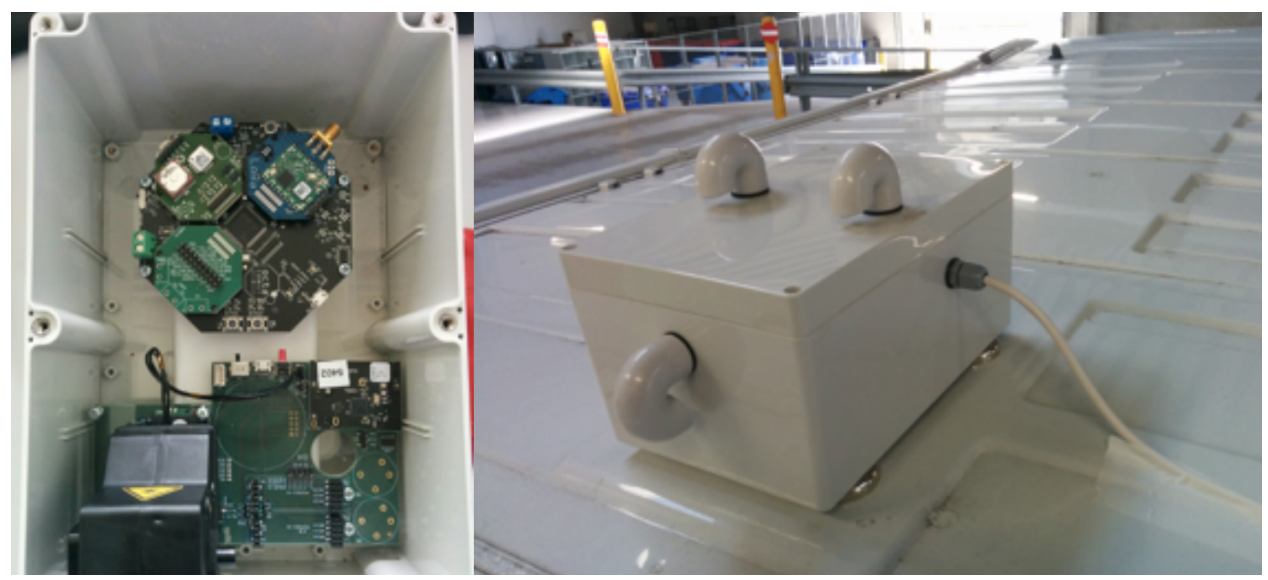

Fig. 4: As part of the Antwerp's City of Things testbed, multi-radio air quality sensors have been mounted on cars of the Belgian postal service.

execution of integrated IoT experiments.

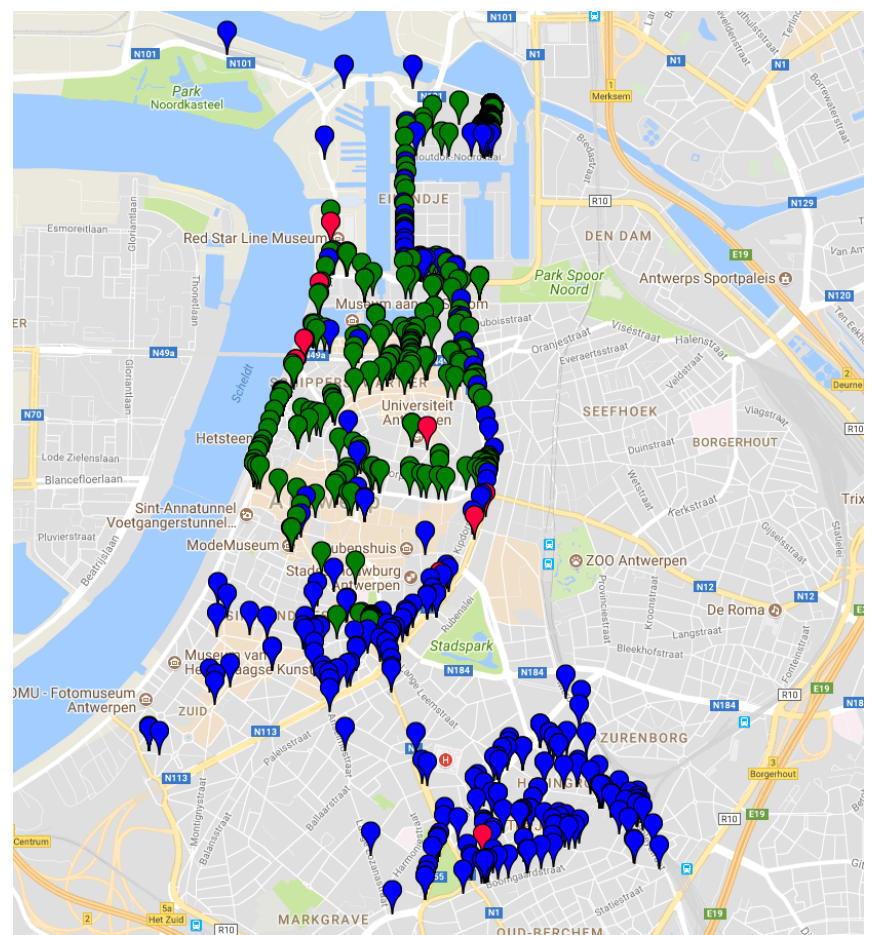

Fig. 5: GPS locations of Bpost cars moving through the city of Antwerp, marked with measurement points on their route (Bpost car 1 - blue; Bpost car 2 - green; Outliers detected by the RC algorithm - red.)

\section{CONCLUSIONS}

In recent years, the need for management functionalities and resource provisioning strategies for Smart Cities is increasing due to the deployment of IoT use cases. Smart Cities aim to provide applications and services based on real-time data retrieved from different devices placed all around the urban area. Proper resource provisioning capabilities are required to minimize resource costs. Therefore, in this article, the CoT framework is presented, which provides not only data collection and analysis operations but also automated resource provisioning mechanisms for Smart City applications. The CoT framework is based on the Tengu platform, an automated orchestration service for the design of custom Big Data frameworks and on LimeDS, a toolkit designed for the rapid development of data-based services. Both Tengu and LimeDS provide suitable components enabling efficient resource provisioning operations in Smart Cities by allowing the design and deployment of specific IoT service models without any kind of manual intervention. Furthermore, the CoT framework allows researchers and stakeholders to access, analyze and process data retrieved from sensors deployed in the city, which results in a high involvement of citizens and companies in the design and development of new applications and services. A Smart City use case based on Air Quality Monitoring through the deployment of air sensors in moving cars has been presented showing the full applicability of the CoT framework in the Smart City environment. As future work, query pre-processing and caching strategies will be developed to avoid large latencies in the processing of massive datasets in near real-time. Furthermore, the integration of Fog Computing concepts, 5G technologies and distributed management approaches are being studied to enhance the architecture of the CoT framework for the future IoT use cases.

\section{ACKNOWLEDGMENT}

The work presented in this article was performed partially within the City of Things project (Antwerp, Belgium) funded by imec and in the "Service-oriented management of a virtualised future internet" project under Grant Agreement \#G059615N, from the fund for Scientific Research-Flanders (FWO).

\section{REFERENCES}

[1] A. Zanella, N. Bui, A. Castellani, L. Vangelista, and M. Zorzi, "Internet of things for smart cities," IEEE Internet of Things journal, vol. 1, no. 1, pp. 22-32, 2014.

[2] V. Albino, U. Berardi, and R. M. Dangelico, "Smart cities: Definitions, dimensions, performance, and initiatives," Journal of Urban Technology, vol. 22, no. 1, pp. 3-21, 2015. 
[3] "Cisco Visual Networking Index: Global Mobile Data Traffic Forecast Update," http://ttp://www.cisco.com/c/en/us/ solutions/collateral/service-provider/visual-networking-index-vni/ mobile-white-paper-c11-520862.pdf (Accessed November 15, 2017).

[4] M. Taneja and A. Davy, "Resource aware placement of iot application modules in fog-cloud computing paradigm," in Integrated Network and Service Management (IM), 2017 IFIP/IEEE Symposium on. IEEE, 2017, pp. 1222-1228.

[5] L. Sanchez, L. Muñoz, J. A. Galache, P. Sotres, J. R. Santana, V. Gutierrez, R. Ramdhany, A. Gluhak, S. Krco, E. Theodoridis et al., "Smartsantander: Iot experimentation over a smart city testbed," Computer Networks, vol. 61, pp. 217-238, 2014

[6] S. Latre, P. Leroux, T. Coenen, B. Braem, P. Ballon, and P. Demeester, "City of things: An integrated and multi-technology testbed for iot smart city experiments," in Smart Cities Conference (ISC2), 2016 IEEE International. IEEE, 2016, pp. 1-8.

[7] T. Vanhove, G. Van Seghbroeck, T. Wauters, F. De Turck, B. Vermeulen, and P. Demeester, "Tengu: An experimentation platform for big data applications." in ICDCS Workshops. IEEE, 2015, pp. 42-47.

[8] S. Verstichel, W. Kerckhove, T. Dupont, B. Volckaert, F. Ongenae, F. De Turck, and P. Demeester, "Limeds and the trapist project: a case study," in 7e International Joint Conference on Knowledge Discovery, Knowledge Engineering, and Knowledge Management (IC3K 2015), vol. 2, 2015, pp. 501-508.

[9] A. Koulakezian, H. M. Soliman, T. Tang, and A. Leon-Garcia, "Robust traffic assignment in transportation networks using network criticality," in Vehicular Technology Conference (VTC Fall), 2012 IEEE. IEEE, 2012, pp. 1-5.

[10] D. Puiu, P. Barnaghi, R. Toenjes, D. Kümper, M. I. Ali, A. Mileo, J. X. Parreira, M. Fischer, S. Kolozali, N. Farajidavar et al., "Citypulse: Large scale data analytics framework for smart cities," IEEE Access, vol. 4, pp. 1086-1108, 2016.

[11] D. P. Abreu, K. Velasquez, M. Curado, and E. Monteiro, "A resilient internet of things architecture for smart cities," Annals of Telecommunications, vol. 72, no. 1-2, pp. 19-30, 2017.

[12] "SLICENET, End-to-End Cognitive Network Slicing and Slice Management Framework in Virtualised Multi-Domain, Multi-Tenant 5G Networks," https://slicenet.eu/ (Accessed November 25, 2017).

[13] R. Petrolo, V. Loscri, and N. Mitton, "Towards a smart city based on cloud of things," in Proceedings of the 2014 ACM international workshop on Wireless and mobile technologies for smart cities. ACM, 2014, pp. 61-66.

[14] "REstore," https://restore.energy/en/homepage (Accessed November 25, 2017).

[15] T. Vanhove, M. Sebrechts, G. Van Seghbroeck, T. Wauters, B. Volckaert, and F. De Turck, "Data transformation as a means towards dynamic data storage and polyglot persistence," International Journal of Network Management, vol. 27, no. 4, 2017.

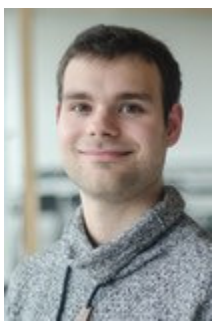

José Santos obtained his M.Sc. degree in Electrical and Computers Engineering in July 2015 from University of Porto, Portugal. He is currently a $\mathrm{PhD}$ Student of Computer Science in the Internet Technology and Data Science Lab (IDLab) Research Group at Ghent University - imec, Belgium. His research interests include Cloud Computing, IoT and Software-Defined Networking. Before joining IDLab, he was a Research Intern at PROEF Group where he was involved in EU-funded projects.

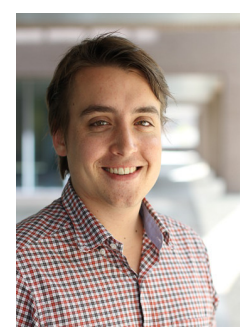

Thomas Vanhove obtained his masters degree in Computer Science from Ghent University, Belgium in July 2012. In August 2012, he started his PhD at the Department of Information Technology (INTEC) at Ghent University, researching data management solutions in cloud environments. More specifically, he has been looking into dynamic big data storages and polyglot persistence. It was during that time he created the Tengu platform for the simplified setup of big data analysis.

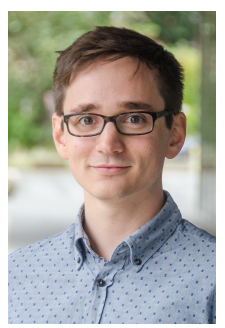

Merlijn Sebrechts graduated in July 2015 as an Industrial Engineer, Informatics, from Ghent University. In August 2015, he joined the Information Technology (INTEC) department of Ghent University to pursue his $\mathrm{PhD}$. In his $\mathrm{PhD}$ he focusses on cloud modelling languages to solve big data challenges, while remaining an active member of several large open-source communities.

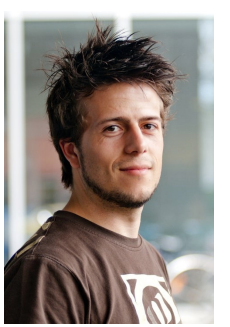

Thomas Dupont is a software engineer in the Department of Information Technology (INTEC) at Ghent University and a senior researcher at IMEC In 2009, he obtained his Master of Science Computer Science Engineering, after which he immediately started working as a full-time researcher at the Faculty of Engineering and Architecture of Ghent University. His research focuses on the design and implementation of distributed middleware architectures.

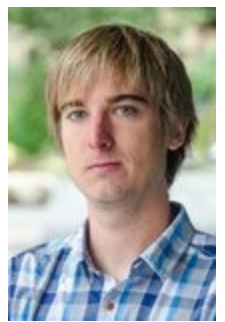

Wannes Kerckhove is a software engineer in the Department of Information Technology (INTEC) at Ghent University and a senior engineer at IMEC. In 2009, he obtained his Master of Science Computer Science Engineering, after which he immediately started working as a full-time researcher at the Faculty of Engineering and Architecture of Ghent University. His research focuses on the design and implementation of scalable software systems.

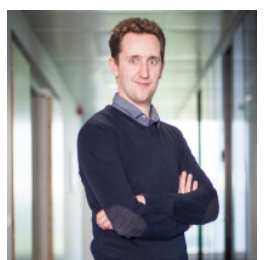

Bart Braem got his Master's degree in Computer Science at the University of Antwerp (magna cum laude). In September 2005, he joined the IDLab research group at the University of Antwerp, where he defended his $\mathrm{PhD}$ thesis on wireless body area networks. Currently a senior researcher at imec in the IDLab research group, he is continuing research on chaotic networks while working on European and regional projects in the smart cities domain. 


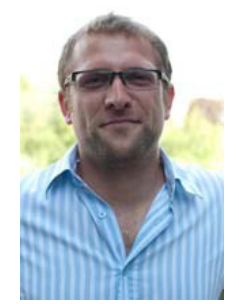

Gregory Van Seghbroeck graduated at Ghent University in 2005. After working as an IT consultant, he joined the Department of Information Technology (INTEC) at Ghent University. In January 2007, he received a $\mathrm{PhD}$ grant from IWT to work on theoretical aspects of advanced validation mechanism for distributed interaction protocols and service choreographies. In 2011 he received his Ph.D. in Computer Science Engineering and continued to work at Ghent University as a post-doctoral fellow.

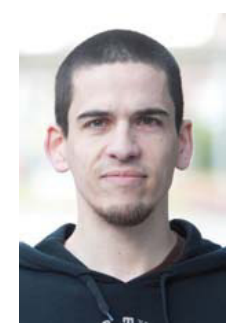

Tim Wauters obtained his M.Sc. and Ph.D. degrees in electro-technical engineering from Ghent University in 2001 and 2007 respectively. He has been working as a post-doctoral fellow in the Department of Information Technology (INTEC) at Ghent University, and is now also active as a senior researcher at imec. His main research interests focus on network and service architectures for multimedia delivery services. His work has been published in about 80 scientific publications.

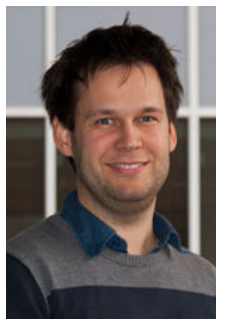

Philip Leroux obtained his masters degree in computer science from Ghent University, Belgium after which he started working as research engineer affiliated with the Department of Information Technology of Ghent University. In 2007, he started a Ph.D. about the optimization of interactive personalized services, which was finished in 2012. Since then he is working as project coordinator and postdoctoral researcher in the context of innovation projects for the (Flemish) media industry.

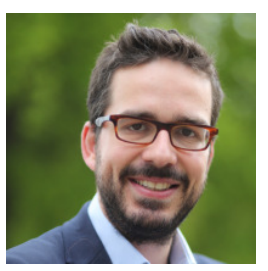

Steven Latré is an assistant professor at the University of Antwerp and imec, Belgium. He is leading the IDLab Antwerp research group, which is performing research in the area of communication networks and distributed systems. He received a Ph.D. in Computer Science Engineering from Ghent University, Belgium with the title "Autonomic Quality of Experience Management of Multimedia Services". $\mathrm{He}$ received the the IEEE COMSOC award for best $\mathrm{PhD}$ in network and service management 2012.

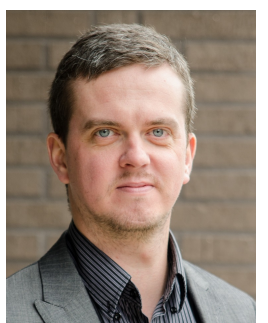

Bruno Volckaert is a professor in software engineering in the Department of Information Technology (INTEC) at Ghent University and senior researcher at imec. He obtained his $\mathrm{PhD}$ at Ghent University on data intensive scheduling and service management for Grid computing in 2006. He has worked on over 35 national and international research projects and is author or co-author of more than 90 papers published in international journals and conference proceedings.

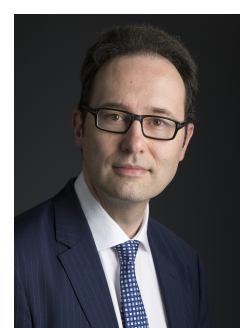

Filip De Turck leads the network and service management research group at the Department of Information Technology of the Ghent University, Belgium and imec. He (co-) authored over 450 peerreviewed papers and his research interests include the design of efficient virtualized network and cloud systems. He serves as Chair of the IEEE Technical Committee on Network Operations and Management (CNOM), and is on the TPC of many network and service management conferences. 